\title{
Ligand-Related Precursors of Luminescent Carbon Nanofibers Synthesized by Microwave Reaction
}

Clara Deeney ${ }^{1}$, Eoin P. McKiernan ${ }^{1}$, Samir A. Belhout ${ }^{1}$, Brian J. Rodriguez ${ }^{2}$, Gareth Redmond ${ }^{1, *}$ and Susan J. Quinn ${ }^{1, *}$

\begin{abstract}
Luminescent carbon nanomaterials are important materials for sensing, imaging, and display technologies. This work describes the use of microwave heating for the template-assisted preparation of luminescent carbon nanofibers (CNFs) from the reaction of a range of beverage-related precursors with the nitrogen-rich polyethyleneimine. Highly luminescent robust carbon fibers that were 10 to $30 \mu \mathrm{m}$ in length and had a diameter of $200 \mathrm{~nm}$ were obtained under moderate conditions of temperature $\left(250-260^{\circ} \mathrm{C}\right)$ and a short reaction time $(6 \mathrm{~min})$. The high aspect ratio fibers showed wavelength-dependent emission that can be readily imaged using epifluorescence. The development of these multi-emissive one-dimensional (1D) carbon nanomaterials offers potential for a range of applications.
\end{abstract}

\section{Keywords:}

carbon fiber; luminescent; carbon nanofiber; beverage; templated synthesis; microwave

\section{Introduction}

In the past 15 years, microwave irradiation has emerged as a powerful tool for the synthesis and functionalization of carbon nanomaterials [1]. This has included the microwave synthesis of carbon nanotubes [2,3], graphitic hollow carbon nanospheres [4], and the exfoliation of graphite to yield graphene [5]. Microwave irradiation has also been used to introduce functional groups at the surface of carbon nanotubes [6], nanohorns [7], and graphene oxide [8]. The key advantage of microwave heating compared to other methods is the access to rapid contactless direct heating that can be switched on or off instantaneously. The use of microwave methods in this way has provided alternatives to harsh reagents, such as acids, allowed the selective heating of reagents, and greatly reduced the reaction times [1].

Luminescent carbon nanomaterials have been widely investigated for their applications in imaging, displays, and sensing. Luminescent carbon dots (CDots), which typically have sizes below $10 \mathrm{~nm}$, have attracted significant interest in recent years due to their biocompatibility and tunable optical properties, with emission observed across the visible spectrum $[9,10]$. These nanoparticles are routinely prepared via the carbonization of a wide range of small molecule precursors, including bio-organic acids, sugars, alcohols, and foodstuffs $[11,12,13,14,15,16,17]$. Furthermore, CDots have also been extracted from commercially available foods and beverages, including pilsner beer [18], Nescafé Original instant coffee [19], and baked goods [13]. Generally, the methods used to prepare CDots include laser ablation, solvothermal synthesis, electrochemical methods, and heating under reflux $[20,21,22,23]$. However, microwave synthesis has proven extremely effective for CDot synthesis with over 900 reports in the literature since 2003. The widespread application of microwave heating for CDots is due to the ready access to carbonization under conditions of rapid heating and increased pressure [15,24,25].

Microwave heating has also been used to prepare carbon fibers [26]. These materials are of interest due to their potential for energy storage as coatings and as catalyst supports $[27,28,29]$. The ability to prepare lightactivated and surface-responsive carbon fibers is expected to expand this capability through potential application 
as environmental sensors and improved coatings. We recently reported the first synthesis of luminescent carbon nanofibers and demonstrated their application for the sensing of metal ions [30]. This was achieved by employing a templated microwave synthesis to perform the reaction of citric acid and polyethyleneimine (PEI); the microwave synthesis of CDots using these precursors had previously been reported. By translating this reaction to a membrane template, rapid microwave heating was found to result in the formation of blue-emitting nanofibers, which could be isolated in solution following the base digestion of the membrane template, as shown in Scheme 1. Shortly after this, improved carbon nanotube growth inside an anodic aluminum oxide template using microwave radiation was also reported [31], which further demonstrates the potential of combining templated synthesis and microwave heating. The mechanism of the formation of the luminescent carbon fibers involved the microwaveassisted hydrothermal carbonization of the precursors. The structure of the template will influence the overall dimensions of the materials, while the material features are expected to be influenced by the temperature and the nature of the molecular precursors. The choice of precursor has been found to influence the optical properties of luminescent CDots with nitrogen containing molecules including urea, ethylene diamine, and polyethyleneimine, resulting in improved luminescence [16,32]. In this report, we now demonstrate the general applicability of the template-assisted microwave method to the preparation of luminescent nanofibers from the reaction of a range of beverage-related precursors with PEI, and describe their luminescent optical properties.

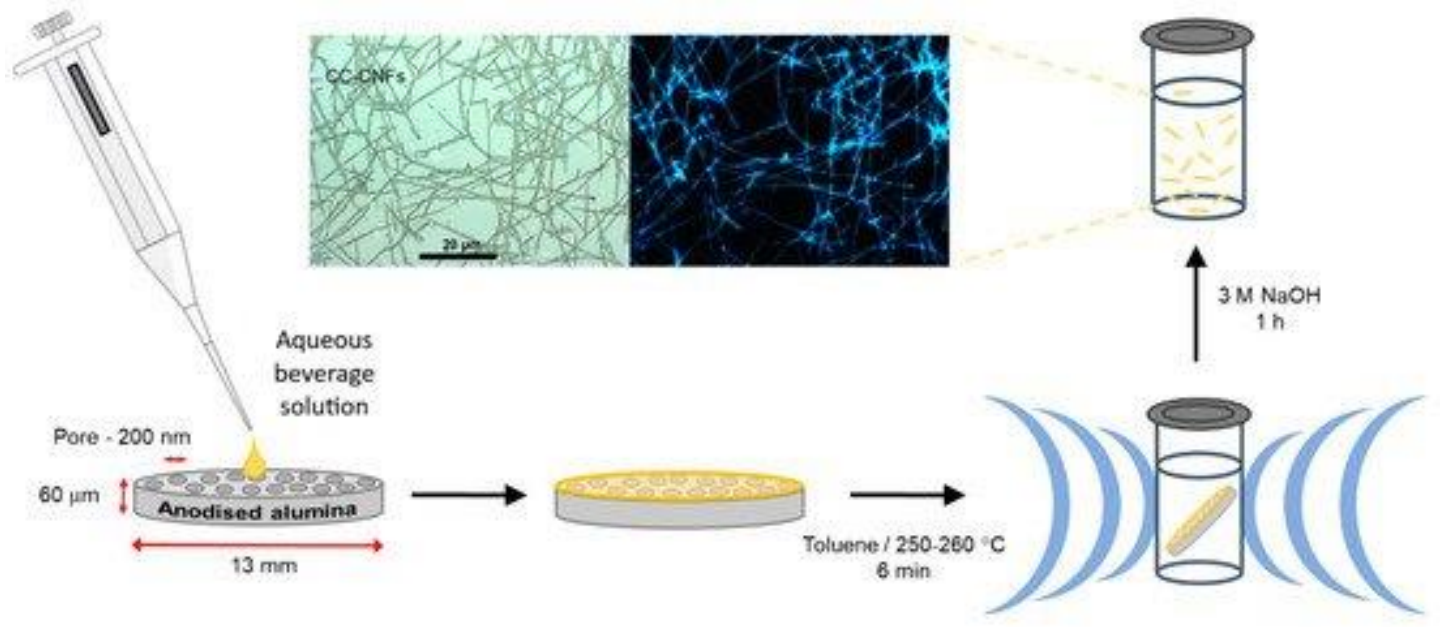

Scheme 1. Overview of the synthesis of luminescent carbon nanofibers via the microwave-assisted hydrothermal pyrolysis of beverage precursors.

\section{Results and Discussion}

Inspired by the literature on CDots, a range of food/beverage-related precursors were chosen to be investigated. These included malic acid (a constituent of apples), which has recently been used to prepare CDots for super-resolution imaging applications [33], and the related lactic acid (milk) for comparison. Four beverages were also considered, including lemon juice, orange juice, and Coca-Cola, all of which have been used to prepare CDots $[12,34,35]$. In addition, grapefruit juice was chosen, which has not yet been reported in the synthesis of CDots. The structures of some of the key molecular components of these beverages together with malic and lactic acid are shown in Scheme 1.

\subsection{Carbon Nanofibers Prepared from Malic Acid (MA-CNF)}

The template-assisted method that was previously developed for the preparation of luminescent citric acid CNFs [30] was first investigated for an aqueous solution of malic acid in the presence of PEI (800 MW). The precursor solution was deposited onto an anodized alumina filter membrane with a 200-nm nominal pore diameter and $60-\mu \mathrm{m}$ length, and allowed to load by gravity over approximately $30 \mathrm{~min}$. The membrane was then transferred 
to a glass reaction vial containing $2 \mathrm{~mL}$ of toluene, which was sealed and subjected to microwave heating at $250{ }^{\circ} \mathrm{C}$ under 16 bar pressure for $6 \mathrm{~min}$. After the reaction, the color of the membrane was found to have changed from a white to a brownish color, as shown in Figure S1. The reaction of the MA was found to require a higher temperature than that used to previously prepare CNFs from citric acid and PEI $\left(200^{\circ} \mathrm{C}\right)$. Removal of the template using $3 \mathrm{M}$ of aqueous $\mathrm{NaOH}$ and washing by dialysis against water gave a percentage yield of $8.4 \pm 2.4 \%$. The yield was calculated based on the dimensions of the membrane template and assuming a CNF density of amorphous carbon of $2.0 \mathrm{~g} / \mathrm{cm}^{3}$, which has been used for the solvent-based synthesis of CDots and CNFs under similar temperature conditions [36,37]. Bright-field images under 100x magnification revealed the successful formation of carbon nanofibers (MA-CNFs) as shown in Figure 1A, which were found to emit blue luminescence when excited between $365-420 \mathrm{~nm}$, as shown in Figure 1B. Analysis of the brightfield images using ImageJ software revealed the average nanofiber length to be $10 \pm 4 \mu \mathrm{m},(n=600)$, with a persistence length of $8 \pm 3 \mu \mathrm{m},(n=650)$, as shown in Figure $1 \mathrm{C}$. The difference in the value of the average length and that of the average membrane pore length of $60 \mu \mathrm{m}$ may be due to incomplete filling of the pores due to poor wetting or some breakage during the membrane digestion process and subsequent workup. However, the isolated fibers were found to be stable to continuous bath sonication for $1 \mathrm{~h}$, with no apparent increase in fragmentation or damage detected under 100× magnification, as shown in Figure S2. Interestingly, when the synthesis of MA-CNFs was repeated under solvothermal conditions in a bomb reactor at $250^{\circ} \mathrm{C}$ for $12 \mathrm{~h}$, the fibers were found to be less well defined, with reduced brightness relative to those prepared under microwave irradiation, as shown in Figure S3. This suggests that the rapid heating conditions that occur in microwave synthesis are important for the structural integrity and emission intensity of the luminescent nanofibers.
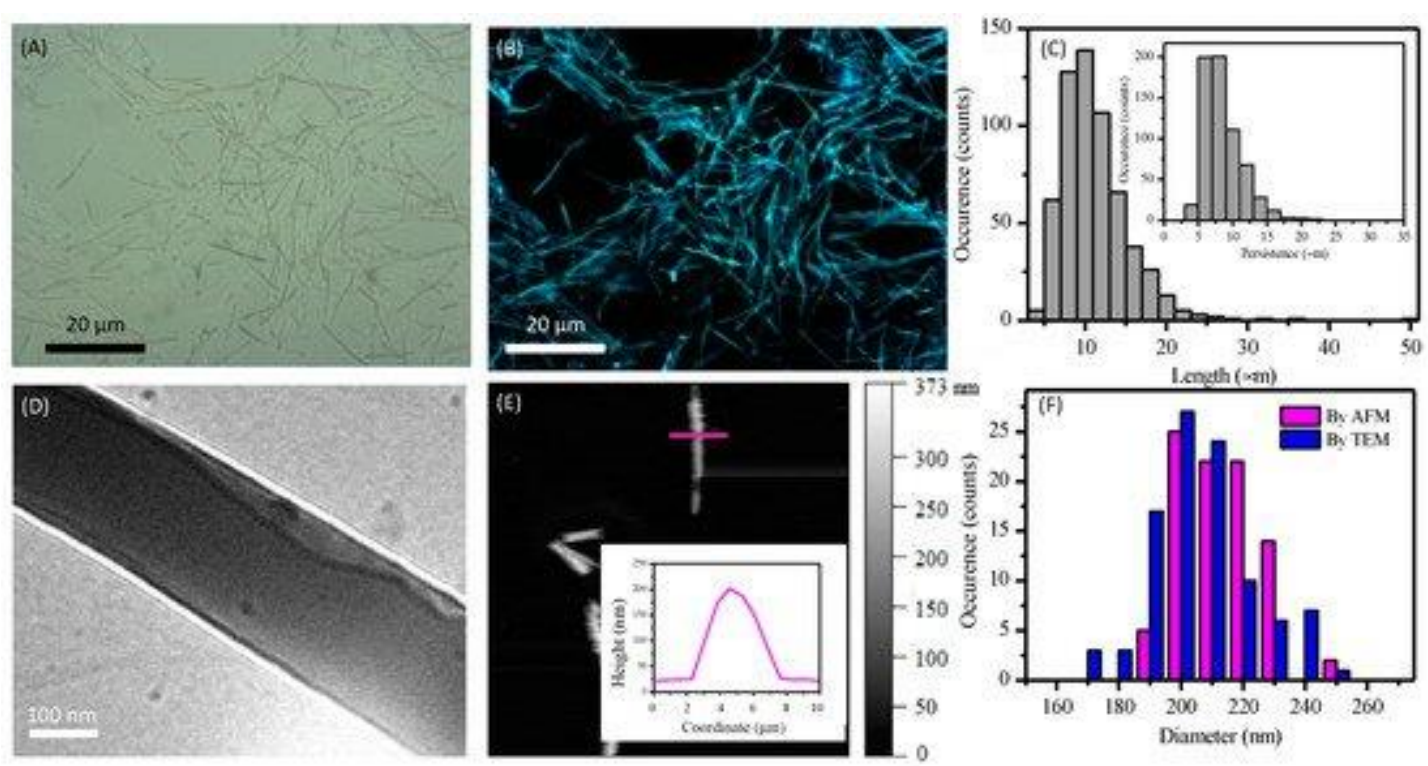

Figure 1. (A) Bright-field image of malic acid carbon nanofibers (MA-CNFs) under 100 $\times$ magnification and (B) corresponding epifluorescence $\left(\lambda_{\text {ex. }} 365-420 \mathrm{~nm} \lambda_{\text {col }}>430 \mathrm{~nm}\right.$ ) image. Drop cast from aqueous suspension onto glass. (C) Length distribution (and persistence length distribution-inset) of MA-CNFs derived from a range of epifluorescence images. (D) TEM image of a MA-CNF on a holey carbon substrate. (E) Atomic force microscopy (AFM) height trace of MA-CNFs in AC mode on glass and inset showing exemplar line trace. (F) Diameter distribution of MA-CNFs by TEM and AFM.

Transmission electron microscopy (TEM) images showed the MA-CNFs edges to be clean with no decoration with smaller particles or frayed edges, as shown in Figure 1D and Figure S4. This suggests that the emission seen in the epifluorescence images is intrinsic to the MA-CNF, and is not a result of aggregated CDots or surface decoration by CDots. The TEM images also show regions of hollows apparently distributed randomly 
through some MA-CNFs. This may be due to the presence of air bubbles in the solution during the synthesis. The presence of these hollows suggests that the preparation of porous luminescent materials may be possible by changing the wetting conditions. The diameter distribution of the cross-section measured by transmission electron microscopy (TEM) taken at 250-nm increments was found to be quite uniform with an average of 201.1 $\pm 27 \mathrm{~nm}(n=100)$, as shown in Figure 1F. Height analysis of the atomic force microscopy (AFM) images yielded an average diameter of $217 \pm 32 \mathrm{~nm}(n=90)$, which is in close agreement with that obtained from the TEM, as shown in Figure 1E,F. These values are also in good agreement with the average diameter of the membrane pores, which was determined by scanning electron microscopy (SEM) analysis of the empty anodized alumina membrane, which was measured as $213 \pm 31 \mathrm{~nm}(n=1087)$, as shown in Figure S5. A summary of the dimensions of the MA-CNF determined using different methods is given in Table S1.

Infrared characterization of the MA-CNFs showed stretching bands for $\mathrm{N}-\mathrm{H}$ at $3361 \mathrm{~cm}^{-1}$ and the acid $\mathrm{O}-\mathrm{H}$ at $2850 \mathrm{~cm}^{-1}$ with bands due to $\mathrm{C}=\mathrm{O}, \mathrm{COO}-$, and the imine $\mathrm{C}=\mathrm{N}$ stretch observed at $1670 \mathrm{~cm}^{-1}, 1390 \mathrm{~cm}^{-1}$, and $1640 \mathrm{~cm}^{-1}$ respectively; see Figure 2A. X-ray photoelectron spectroscopy (XPS) was used to determine the chemical composition of the MA-CNFs, which was found to be $62.0 \%$ carbon, $11.0 \%$ nitrogen, and $27 \%$ oxygen. The high-resolution analysis of the XPS data was used to provide information on surface functionality. Three peaks were recorded at $286 \mathrm{eV}, 401 \mathrm{eV}$, and $532 \mathrm{eV}$ corresponding to the $\mathrm{C}$ 1s peak, $\mathrm{N}$ 1s peak, and $\mathrm{O}$ 1s peak, respectively (Figure $2 \mathrm{~B}$ ), which are similar to those previously recorded for CDots prepared from malic acid $[33,38]$. The $\mathrm{C}$ 1s peak is composed of contributions from $\mathrm{C}-\mathrm{O} / \mathrm{C}-\mathrm{C}$ at $286.1 \mathrm{eV}$ and $\mathrm{C}=\mathrm{O}$ at $288.9 \mathrm{eV}$; see Figure $2 \mathrm{C}$. In contrast to the XPS spectra previously reported for the fibers prepared using citric acid, the presence of $\mathrm{C}-\mathrm{C} / \mathrm{C}=\mathrm{C}$ was not observed in the XPS spectrum, although it is expected to contribute weakly to the envelope. A similar reduction in the $\mathrm{C}=\mathrm{C}$ contribution has been observed for the XPS spectra recorded for Cdots prepared from malic acid as compared to citric acid; see Figure $2 \mathrm{C}$ [38]. The $\mathrm{N}$ 1s peak indicates the presence of pyrrolic $(400.3 \mathrm{eV})$ and graphitic $\mathrm{N}(401.3 \mathrm{eV})$, while the $\mathrm{O} 1 \mathrm{~s}$ peaks at $532.4 \mathrm{eV}$ and $533.2 \mathrm{eV}$ are assigned to $\mathrm{C}=\mathrm{O}$ and ester-like O-C groups; see Figure 2D,E $[39,40,41]$. The slightly higher value in the $C=O$ band assignment here compared to that reported for Cdots prepared from carbohydrates [39] is likely due to the a contribution from C-O species as previously observed [41] The Raman spectrum recorded for the fibers shows no defined D or $G$ band signal, and combined with no apparent lattice structure in TEM, it suggests that the carbon composition of the MA-CNF is amorphous in nature; see Figure S6 [42].
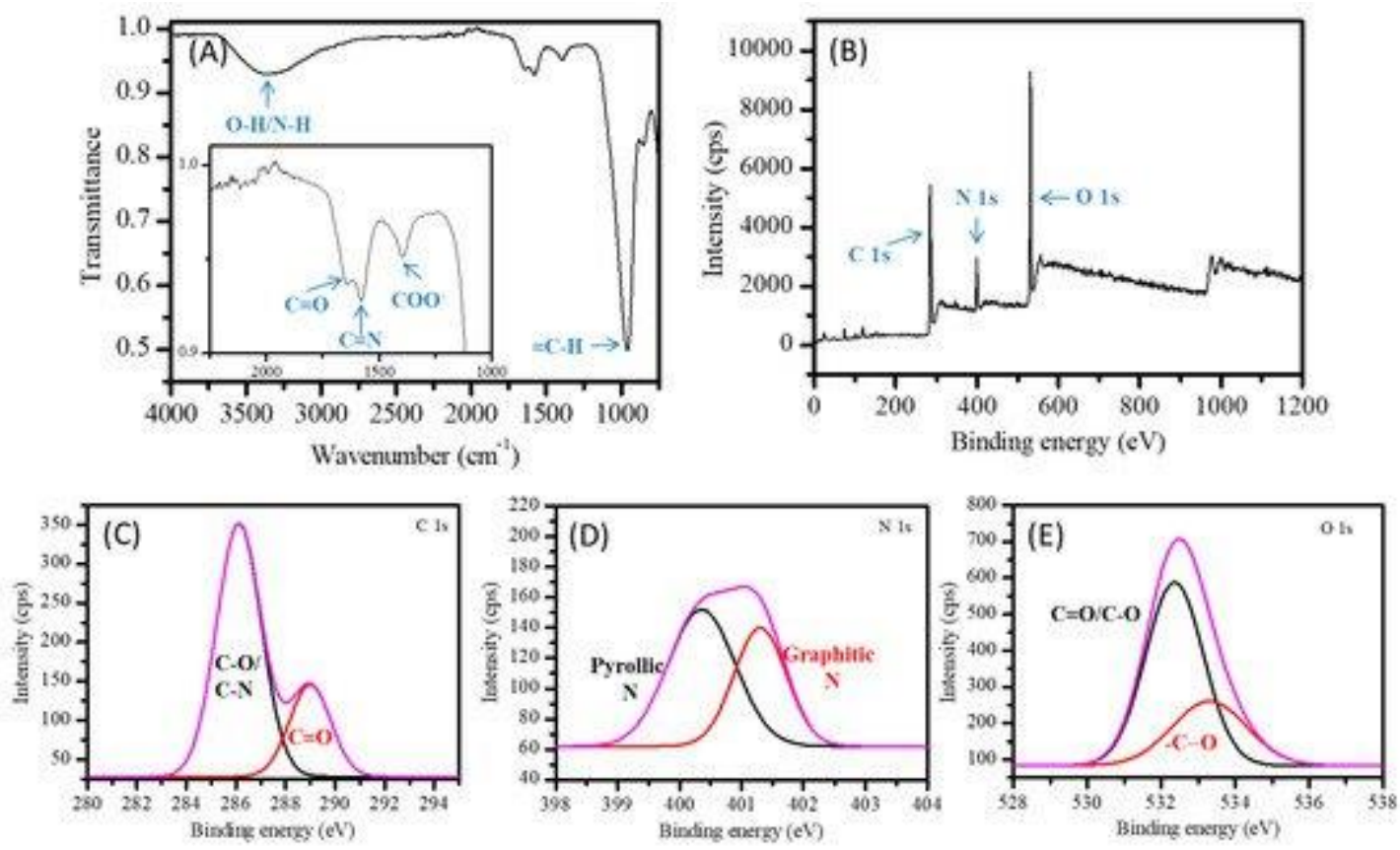
Figure 2. (A) Solid state Fourier transform infrared (FTIR) spectrum of MA-CNFs dried under reduced pressure. (B) X-ray photoelectron spectroscopy (XPS) full-range spectrum of MA-CNFs drop cast sequentially from aqueous suspension. (C-E) High-resolution XPS spectra of C 1s, N 1s, and O 1s regions, respectively.

The MA-CNFs were found to have an unstructured absorption across the visible region of the spectrum, as shown in Figure 3A. Excitation of the sample at $345 \mathrm{~nm}$ resulted in a broad emission (full-width half-maximum, or FWHM $=145 \mathrm{~nm}$ ) centered at $455 \mathrm{~nm}$, which is greater than that previously obtained for citric acid CNFs; see Figure S7 [30]. The excitation spectrum showed a maximum of $340 \mathrm{~nm}$, which represents a significant Stokes shift of $115 \mathrm{~nm}$. The emission of the MA-CNF was found to be highly dependent on the excitation wavelength with the emission at $500 \mathrm{~nm}$ detected upon visible light excitation; see Figure 3B. This observation of excitation wavelength-dependent emission is in contrast to the largely excitation-independent emission seen in CA-CNFs, which is characteristic of molecular based emission $[43,44]$. This suggests that there may be multiple molecular-like species contributing to the emission $[45,46]$. The quantum yield of MA-CNFs was found to be $2.7 \%$ by integration sphere, which is notably lower than that of CA-CNFs $(4.8 \%)$ and lower than CDots made from bio-organic acid and amine precursors [16]. The data obtained from fluorescence lifetime measurements fitted well to a biexponential decay with a short-lived species of $1.4 \mathrm{~ns}$ and a contribution from a longer-lived component at $7.7 \mathrm{~ns}$. The broad (blue to yellow) wavelength-dependent emission was readily captured by recording the epifluorescence images under different conditions of excitation and collection; see Figure 3D and Figure S8.
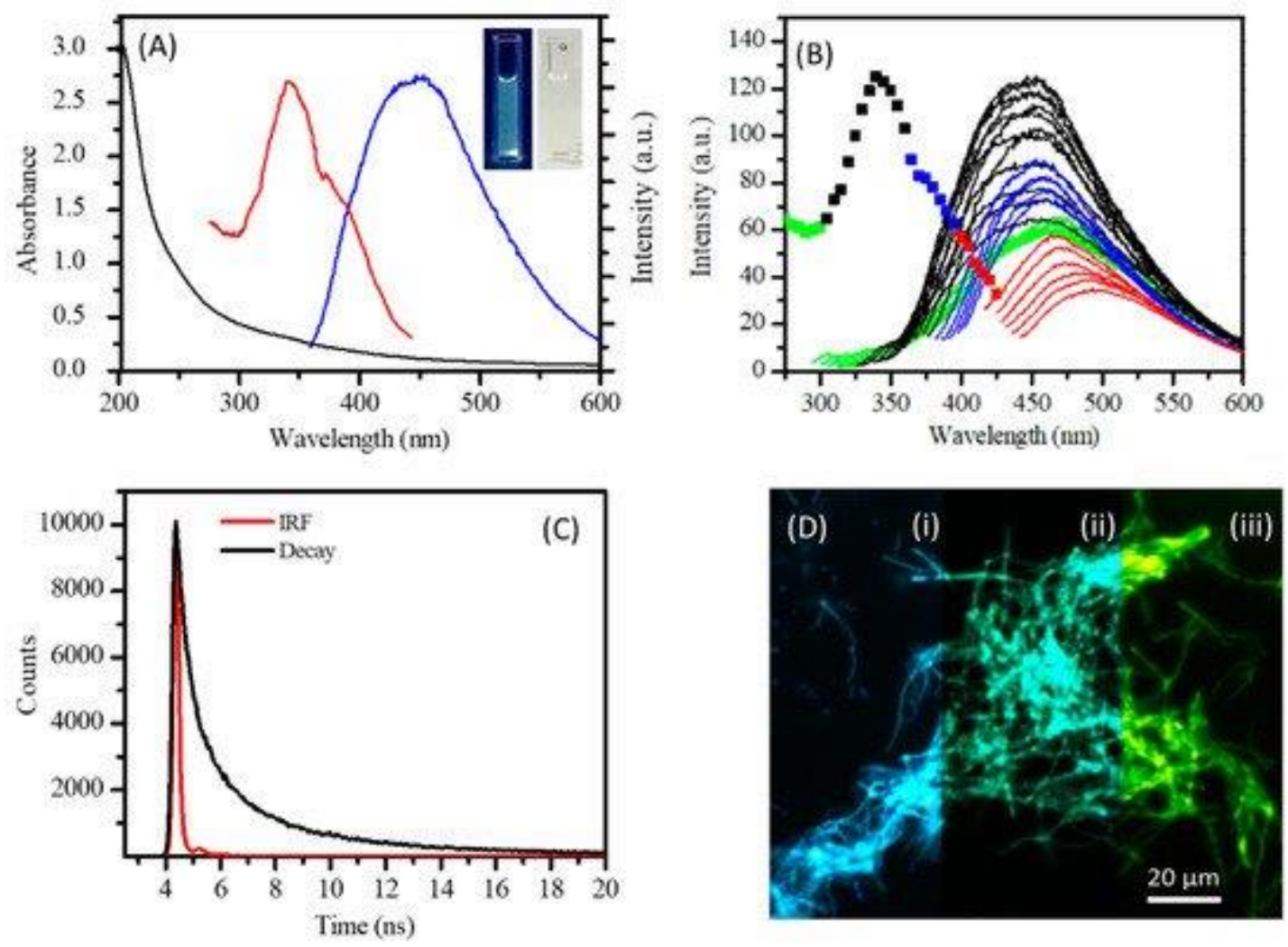

Figure 3. (A) Absorbance (black) emission at $\lambda_{\mathrm{ex}} 345 \mathrm{~nm}$ (blue) and excitation at $\lambda_{\mathrm{em}} 455 \mathrm{~nm}$ spectra of MA-CNFs in aqueous suspension. Inset shows suspension under 365-nm illumination and ambient lighting. (B) Excitation wavelength-dependent emission spectra of MA-CNFs at 5-nm increments from $\lambda_{\text {ex }} 274 \mathrm{~nm}$ to $425 \mathrm{~nm}$ ( $\lambda_{\text {ex: }}$ 275$300 \mathrm{~nm}$ green, 305-360 nm black, 365-390 nm blue, and 395-425 nm red) and the associated extrapolated excitation spectrum in squares. (C) Fluorescence lifetime decay of MA-CNFs in aqueous suspension $\lambda_{\text {ex }} 365 \mathrm{~nm}$ $\lambda_{\text {col }} 455 \mathrm{~nm}$. (D) Epifluorescent images captured under (i) $\lambda_{\text {ex. }} 365-420 \mathrm{~nm}, \lambda_{\text {col }}>430 \mathrm{~nm}$; (ii) $\lambda_{\text {ex. }} 390-407 \mathrm{~nm}, \lambda_{\text {col }}>$ $407 \mathrm{~nm}$; and (iii) $\lambda_{\text {ex. }} 450-490 \mathrm{~nm}, \lambda_{\text {col }}>490 \mathrm{~nm}$. 
The surface state nature of CDot emission is typically sensitive to the surrounding environment and has been exploited for numerous sensing applications $[47,48,49]$. The MA-CNF emission was found to be sensitive to $\mathrm{pH}$ with changes in both intensity of emission (up to $60 \%$ ), and the position of the $\lambda_{\max }$ (up to $20-\mathrm{nm}$ redshift from $455 \mathrm{~nm}$ ) observed; see Figure S9A [50,51]. The emission response was found to be reversible upon cyclization from $\mathrm{pH} 7$ to $\mathrm{pH} 1$ to $\mathrm{pH} 13$, which suggests the role of protonation and deprotonation of surface groups; see Figure S9B. The treatment of MA-CNFs with sodium borohydride caused a shift in the wavelength of maximum emission from $455 \mathrm{~nm}$ to $425 \mathrm{~nm}$ with an increase in the intensity of $155 \pm 15 \%$; see Figure S10.

Similar changes have been observed upon $\mathrm{CDot}$ reduction and were attributed to the reduction of $\mathrm{C}=\mathrm{O}$ and $\mathrm{C}=\mathrm{N}$ functional groups [46]. The results from these two studies serve to demonstrate the ability to modulate the emission of these CNFs. The method to prepare MA-CNFs was also successfully used to prepare luminescent CNFs by reacting lactic acid and PEI under identical conditions of microwave synthesis and purification; see Tables S3 and S4. These nanofibers exhibited very similar properties to the MA-CNFs; see Figure S11.

\subsection{Carbon Nanofibers Prepared from Commercially Available Beverages}

Having prepared CNFs from the molecular constituents of citrus fruit (citric acid) [30], apple (malic acid), and milk (lactic acid), we next considered whether the use of available foodstuffs could be used. This study investigated the use of orange juice (Oj), grapefruit juice $(\mathrm{Gj})$, lemon juice $(\mathrm{Lj})$, and the soft drink Coca-Cola (CC). The relationship between the chemical structure and luminescence properties of Cdots is not yet fully resolved [16,20], with the origin of the observed luminescence expected to be derived from a combination of surface molecularbased species and the core carbon state [16]. In particular, the influence of the population of amines at the surface has been observed to increase the wavelength of emission from blue to green [17]. The use of water-soluble precursors with hydrophilic groups is expected to influence the nature of the surface of the carbon fibers, which may be tuned by varying the temperature and ratio of amine-containing groups. As such, the luminescence properties of the microwave-assisted hydrothermal carbonization of readily available beverage precursors is expected to be influenced by: (a) the major constituents of the beverages, including sucrose, glucose, fructose, citric acid, and ascorbic acid, as well as the more conjugated furanocoumarin and caffeine, and (b) the presence and absence of PEI. To do this, a 10X juice/soft drink concentrate (by evaporation) was reacted (i) with and (ii) without PEI at $260{ }^{\circ} \mathrm{C}$ for $6 \mathrm{~min}$; see Tables S3 and S4. In all the cases, the reaction conditions resulted in a darkening of the membrane appearance, which was taken as an indication of the reaction proceeding. The brightfield images of Lj-CNF, Gj-CNF, Oj-CNF, and CC-CNF prepared with PEI revealed highly persistent and relatively long CNFs that showed little fragmentation; see Figure 4A-D. The epifluorescence images showed strong emission for the drinks-based CNFs prepared using PEI; see Figure 4A-D. In contrast the CNFs prepared without PEI (CNF(no-PEI)) were only weakly emissive; see Figure S12. 

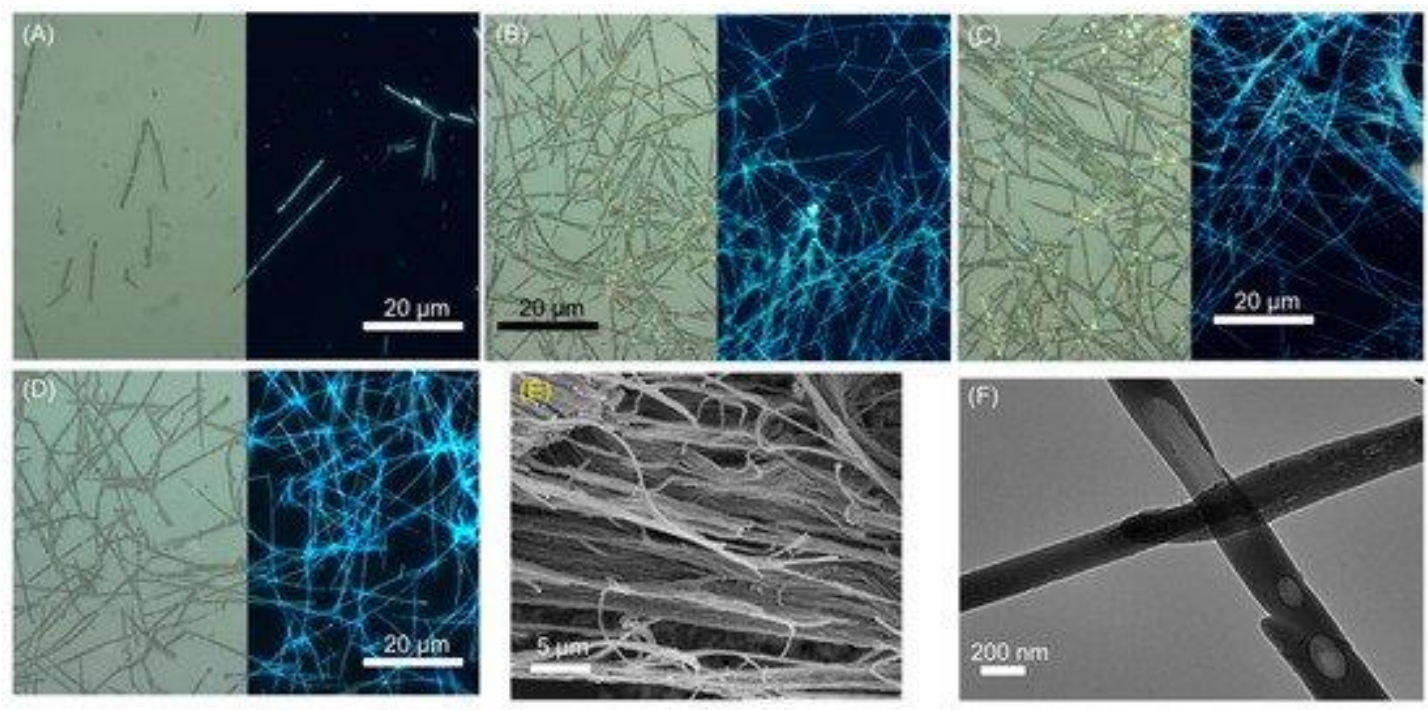

Figure 4. (A-D) 100x mag images of Lj-CNFs, Oj-CNFs, Gj-CNFs and CC-CNFs respectively under conditions of bright-field and epiflourescence illumination ( $\lambda_{\text {ex }} 365-420 \mathrm{~nm}, \lambda_{\text {col }}>430 \mathrm{~nm}$ ). Scale applies to all. (E) SEM image of gold-sputtered CC-CNFs. (F) TEM image of CC-CNFs drop cast from aqueous suspension onto a lacey carbon substrate and dried in air. Lj: lemon juice, Oj: orange juice, Gj: grapefruit juice, CC: Coca-Cola.

The lengths of the drink-CNFs all show a relatively long total length $(17-37 \mu \mathrm{m})$ compared to MA-CNFs (11 $\mu \mathrm{m})$; see Table 1 and Figure S13. The longest CNFs measured were for Oj without PEI and Gj without PEI (32 \pm $11 \mu \mathrm{m}$ and $37 \pm 10 \mu \mathrm{m}$, respectively). Each of the drink-CNFs had instances of nanofibers of $60 \mu \mathrm{m}$, which is the reported full length of the membrane template. This suggests that the reaction mixture is fully filling the template, but importantly, no CNFs longer than $60 \mu \mathrm{m}$ were seen, which implies that the drink-CNFs are being formed inside the template pores and not crystalizing on the surface of the membrane. This is again illustrated by the SEM image of the gold-sputtered membrane after partial digestion for CC-CNF, which shows a dense forest of fibers; see Figure 4E. The TEM image of CC-CNFs show the surfaces to be smooth with no indication of any CDot coating; they also reveal the presence of structural hollows along the lengths of the CNFs, which have been observed for the MA-CNF, as shown in Figure 4F.

Table 1. Average lengths of drink-CNFs as measured by bright-field microscopy.

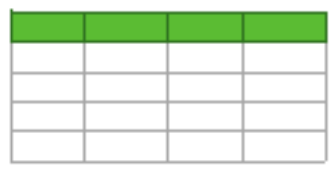

The chemical composition of the drink-CNFs was measured by combustion elemental analysis, which showed that when PEI was used, the $\mathrm{N}$ composition was approximately $13 \%$ and decreased to $<2 \%$ for the CNFs prepared without PEl; see Table S5. These results were reflected in the Fourier transform infrared (FTIR) of the drink-CNFs, which showed a greater contribution to the amide stretch at $1633 \mathrm{~cm}^{-1}$ for samples containing PEI; see Table S6 and Figure S14.

The absorbance spectra of the CNFs prepared with PEI show some structured absorption below $400 \mathrm{~nm}$ with a gradual decrease of absorbance from 400 to $800 \mathrm{~nm}$; see Figure 5A. In contrast, the spectra obtained for the reaction without PEI are largely featureless; see Figure S14. The epifluorescence images revealed the PEI samples to be more emissive, and these samples were found to display excitation-dependent emission; see Figure S16 and Table S7. The maximum emission was recorded at 350-nm excitation, which resulted in emission centered between 455-464 nm, with the greatest emission intensity found for CC-CNF. Notably, the FWHM of $50 \mathrm{~nm}$ of the excitation spectrum is narrower for all the PEI drink-CNFs than the MA-CNF (90 nm), see Figure 5B,C. In all the 
cases, excitation at $400 \mathrm{~nm}$ resulted in weaker green emission centered at ca. $500 \mathrm{~nm}$. In line with the observations made by epifluorescent imaging, the drink-CNFs prepared without PEI were less emissive. Weak luminescence was observed for Oj-CNF(no-PEI) and Gj-CNF(no-PEI) samples, but the Lj-CNF(no-PEI) and CC-CNF(no-PEI) were non-luminescent; see Figure S17. This observation reflects previous reports that indicate the requirement of nitrogen-containing precursors to provide strong emission [11]. A comparative summary of the emission intensity and the fiber lengths of the different systems is given in Figure $6 B, C$, and highlights the improved structural and emissive properties the CC-CNF and Gj-CNF over the MA-CNF sample. As all three CNFs were prepared using $\mathrm{PEI}$, it is reasonable to conclude that the added molecular components present in the fruit juice are important. Interestingly, sugar molecules such as fructose are present in high concentrations in fruit juices, and have recently been used to prepare CDots [49].
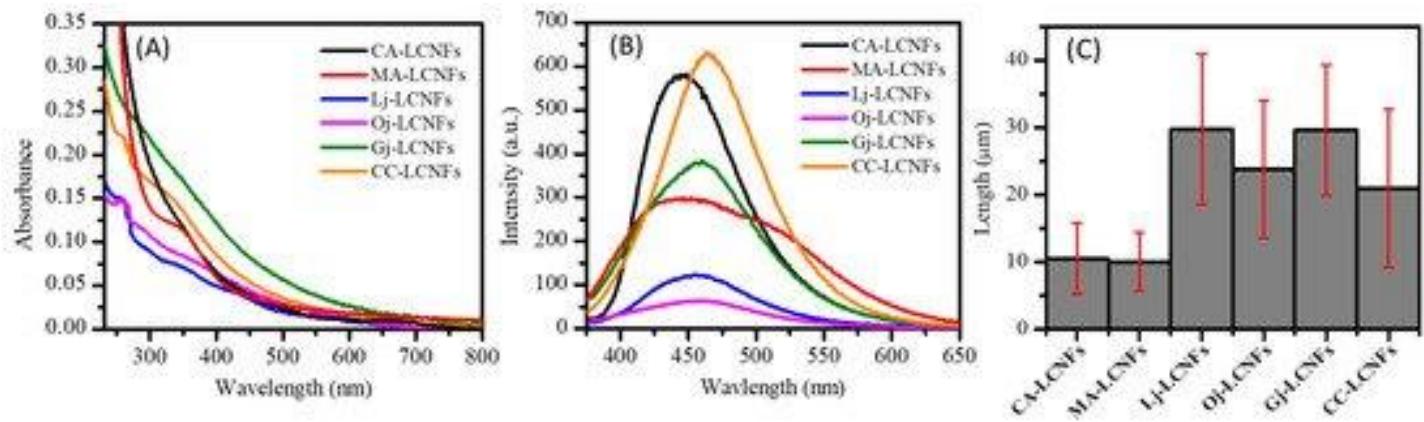

Figure 5. (A) Absorbance spectra of all the CNF samples in aqueous suspension. (B) Emission spectra of all the CNF samples in aqueous suspension $\lambda_{\text {ex }} 350 \mathrm{~nm}$, Abs 0.1 at $350 \mathrm{~nm}$ for all samples. (C) Length comparison of a CNF samples as determined by optical microscopy.
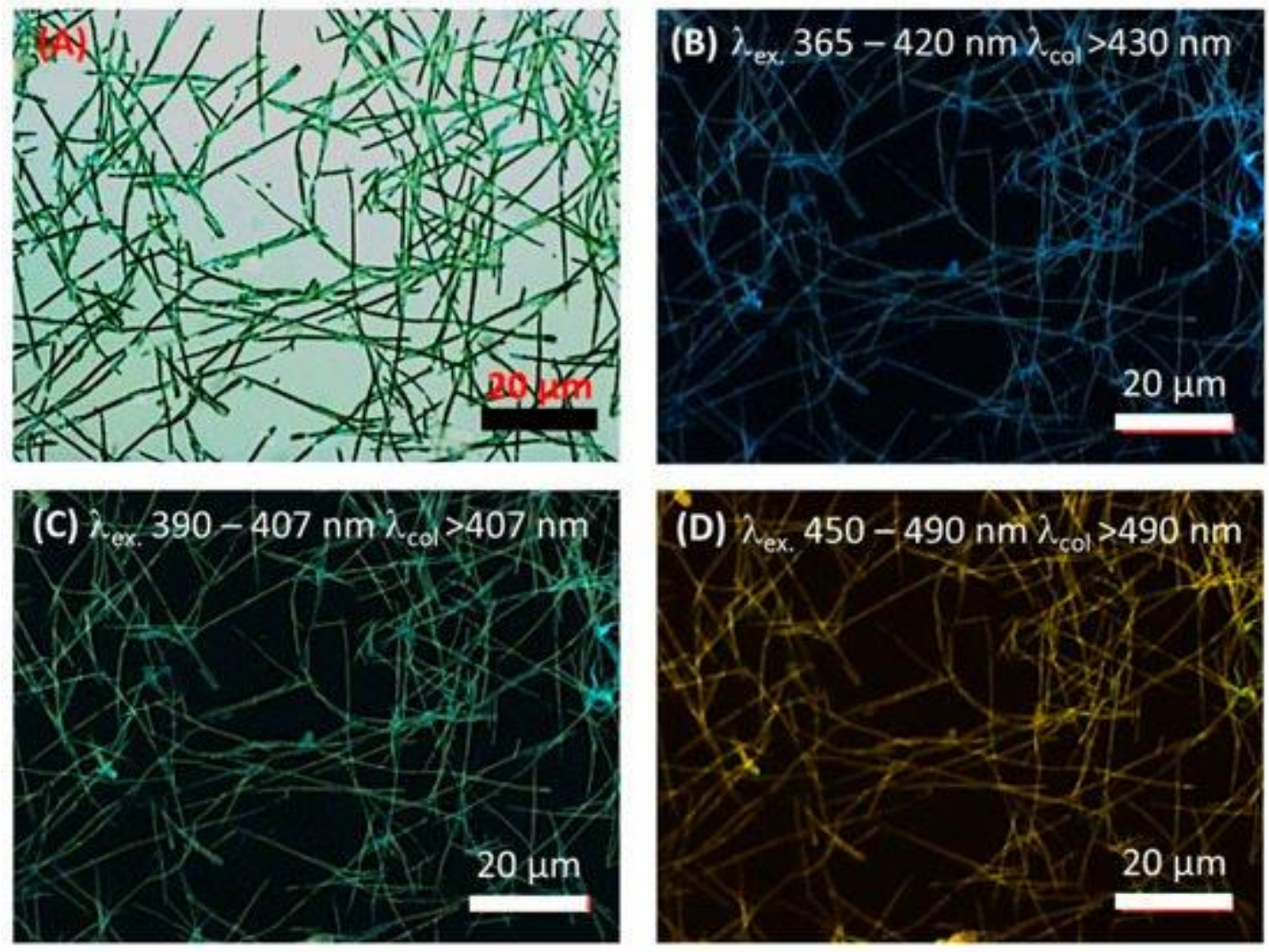

Figure 6. 100x magnified images of CC-CNFs under (A) bright-field illumination and (B-D) epifluorescence illumination as shown.

Finally, the wavelength-dependent emission demonstrated by the drink fibers was also observed under epifluorescence imaging and recorded under different conditions of filtered light excitation and collection, which show the dramatic range of emission from the CC-CNFs; see Figure 6. 


\section{Materials and Methods}

Carbon fibers were prepared by dropping the precursor solution $(40 \mu \mathrm{L})$ onto the surface of a $0.2-\mu \mathrm{m}$ pore diameter Anodisc (anodized alumina) membrane and allowing it to wet the pores for approximately $30 \mathrm{~min}$. The filled membrane was transferred to a 10-mL glass microwave vessel and submerged in $2 \mathrm{~mL}$ of toluene, which had not been degassed, and the vial was sealed with a silicone septum held in place by a PEEK-made cap. The sample was microwaved at $6 \mathrm{~min}$ (see the temperature and pressure conditions in Table S4). The membrane was removed from the toluene and left to dry in air. Any excess solid carbon was removed from the upper and lower surface of the membrane using a blade. Then, the membrane was placed in a 2-mL Eppendorf vial with $1 \mathrm{~mL}$ of $3 \mathrm{M} \mathrm{NaOH}$, and the vial was wrapped in aluminum foil to keep it in the dark while it shook for $1 \mathrm{~h}$ to dissolve the membrane. Sonication was used for $15 \mathrm{~min}$ to separate the nanofibers (not needed for MA-CNFs). Then, the suspension was centrifuged at 11,000 for $3 \mathrm{~min}$, and the supernatant discarded and replaced with deionized water to remove the $\mathrm{NaOH}$; this centrifuge step was repeated five times. In the case of MA-CNF, the suspension was dialyzed in a 3500 MWCO (molecular weight cut-off) membrane in a 2-L water bath for at least $1 \mathrm{~h}$, with 10 water changes. All the samples were stored in aqueous suspension in the dark. The MA-CNF yield was calculated from the experimental weight of the nanofiber sample and the theoretical yield as determined by the number of pores recorded from the SEM image of the empty template $\left(1.84( \pm 0.22) \times 10^{9}\right)$, Figure S5. The volume of the pore $(1.9$ $\left.( \pm 0.23) \times 10^{-18} \mathrm{~m}^{3}\right)$ was valued based on the width determined using a diameter of $237.3 \pm 39.2 \mathrm{~nm}$ and a height of $60 \mu \mathrm{m}$, as specified. The equivalent carbon mass of $8.7 \mathrm{mg}$ was then calculated assuming the nanofibers have the density of amorphous carbon, $2.0 \mathrm{~g} \mathrm{~cm}^{-3}$.

\subsection{Characterisation of Dispersed Carbon Fibers}

The spectroscopic properties of the as prepared aqueous carbon nanofiber aqueous suspension was measured in a low volume $(500 \mu \mathrm{L})$ quartz cuvette. Absorbance was measured on a Cary 60 and excitation and emission were measured on a Cary Eclipse. Emission spectra for suspensions of carbon fibers (absorbance at $350 \mathrm{~nm}$ at 0.1 ) were taken. These carbon fiber suspensions were spun down via centrifugation and resuspended in buffers of known $\mathrm{pH}(\mathrm{pH} 1, \mathrm{pH} 3, \mathrm{pH} 5, \mathrm{pH} 7, \mathrm{pH} 9$ and $\mathrm{pH} 11)$, and the emission spectra was repeated. For emission switching, the emission spectrum was taken a suspension of carbon fibers in water $(\mathrm{pH} 7)$. Conc. $\mathrm{HCl}$ was added resulting in $\mathrm{pH}$ of one (emission spectrum was taken) followed by addition of conc. $\mathrm{NaOH}$ until the $\mathrm{pH}$ had reached 11 (emission spectrum was taken). Lifetime measurements were made on a Jobin Yvon Horiba FluoroCube-01-NL with excitation $365 \mathrm{~nm}$, collection wavelength was $455 \mathrm{~nm}$. The Instrument Response Frequency was found using Ludox colloidal silica in water. Quantum yield measurements. The as prepared aqueous carbon nanofiber aqueous suspension was measured in a $3 \mathrm{~mL}$ quartz cuvette. With absorbance 0.1 at excitation wavelength. The baseline was taken with deionized $\mathrm{H}_{2} \mathrm{O}$ and an integration sphere was used to collect the absorbance and emission counts.

\subsection{Characterisation of Deposited Samples}

AFM images were taken in amplitude modulation mode in air using an Asylum Research MFP-3D with a PPP-NCH tip (Nanosensors, Neuchatel, Switzerland). The instrument was Asylum Research MFP-3D AFM, at a scan rate of $1 \mathrm{~Hz}$. Raman measurements was performed on a SENTERRA dispersive Raman microscope (Brunker optics) with a $785 \mathrm{~nm}$ laser. Settings applied were $1 \mathrm{~mW}$ power, $10 \mathrm{~s}$ collection times and 10 accumulations. The sample was deposited from aqueous suspension onto a cleaned $\mathrm{CaF}_{2}$ plate, a total of $6 \mathrm{~mL}$ as prepared nanofiber was dropped sequentially onto the same area in $10 \mu \mathrm{L}$ aliquots. A nanofiber sample was dried under reduced pressure. The IR spectra were recorded for the dried using a Brunker Alpha-Platinum ATR, with a background acquired in air. X-ray Photon Spectroscopy (XPS) was performed using Kratos Axis Ultra XPS system, Kratos 
Analytical Ltd. (Manchester, UK) using a monochromatic Al K a1,2 source (150 W $15 \mathrm{kV}$ and pass energy $10 \mathrm{eV}$ for the high resolution scans and $160 \mathrm{eV}$ for the wide scan). Spectra were analysed using Vision software (by Kratos). The peak positions were referenced to the $\mathrm{C}$ 1s hydrocarbon peak at $284.8 \mathrm{eV}$ and a linear subtraction was applied. The peaks were fitted using an asymmetric GL(30) (Gaussian/Lorentzian product). The sample holder was sonicated in ethanol for $30 \mathrm{~min}$ and dried in air to clean. $10 \mu \mathrm{L}$ of the carbon fiber suspension at a time were deposited into the middle of the sample holder and dried in air at $60{ }^{\circ} \mathrm{C}$. This was repeated until a total of $3 \mathrm{~mL}$ was deposited and dried to ensure no gaps between the fibers, revealing the sample holder.

SEM measurements were taken using a FEI Quanta 3D FEG DualBeam (FEI Ltd., Hillsboro, OR, USA). Once the membrane was removed from the microwave and dried in air, the 'top side' was identified (more uniform pore distribution in anodizing process-appears shinier) and this side was scratched only. The underside was stuck to an adhesive carbon pad and approx. $100 \mu \mathrm{L} 3 \mathrm{~mol} \mathrm{L-1} \mathrm{NaOH}$ was dropped onto the membrane and left for $25 \mathrm{~min}$. The membrane (still attached to the pad) was then rinsed with clean deionized $\mathrm{H}_{2} \mathrm{O}$ and left to dry. The carbon pad was then stuck to a sample holder. Samples were sputter coated with gold, using an Emitech K575X Sputter CoatingUnit, to prevent surface charging by the electron beam. Transmission Electron Microscope (TEM) and Scanning Transmission Electron Microscope (STEM) images were recorded on a FEI TITAN TEM and a JEOL2100 TEM at acceleration voltage $300 \mathrm{kV}$. The nanofibers were deposited from aqueous suspension onto a holey carbon substrate and dried in air under desiccation conditions.

\section{Conclusions}

Microwave heating has become an increasingly important method for the development of new carbon nanomaterials. Here, we have demonstrated the general applicability of microwave heating and template synthesis to prepare luminescent carbon fibers from a range of beverage-related precursors. Robust CNFs were obtained under conditions of moderate microwave heating and a short reaction time. The structural quality and emission properties were observed to be improved compared to fibers obtained using the templated under solvothermal synthesis conditions. The CNFs prepared from fruit juice and Coca-Cola showed significant improvements in persistence length and the emission properties over those prepared from malic and lactic acid, which may arise due to the presence of sugar molecules. Interestingly, the use of a nitrogen-rich polyimine proved critical to ensure good luminescent, and future work will consider the role of other nitrogen containing precursors such as urea. The CNFs prepared were found to display excitation-dependent emission spanning from blue to green. Future work will consider the ability to tune this emission to access red-emitting materials. TEM measurements revealed pores in the CNF structures. The ability to tune the density of these may allow the development of porous luminescent carbon materials for sensing, as the MA-CNFs luminescence was found to be sensitive to $\mathrm{pH}$, and CA-CNFs have been shown to be capable of metal ion sensing [30]. Here, microwave heating treatment of the solid material may be investigated, as this has been previously used to generate porous material from carbon solid precursors [1]. Going forward, the carbon luminescent fibers reported here may be incorporated into sensing devices to report on environmental changes, such as changes in $\mathrm{pH}$ and composite materials to act as catalyst supports.

\section{References}

1. Shen, F. Z.; He, F.; Lu, D.; Xie, Z. Q.; Xie, W. J.; Ma, Y. G.; Hu, B., Bright and colour stable white polymer lightemitting diodes. Semicond Sci Tech 2006, 21, L16-L19.

2. Park, J. J.; Park, T. J.; Jeon, W. S.; Kim, S. Y.; Lee, Y. K.; Jang, J.; Kwon, J. H., White polymeric light-emitting diodes based on doping of an orange ir complex in a fluorene blue polymer. Idw '07: Proceedings of the 14th 
International Display Workshops, Vols 1-3 2007, 1033-1036.

3. Tang, K. C.; Tseng, S. R.; Li, W. S.; Meng, H. F.; Horng, S. F.; Hsu, C. S., Broad band and white phosphorescent polymer light-emitting diodes in multilayer structure. Synthetic Met 2008, 158, 287-291.

4. Park, J. J.; Park, T. J.; Jeon, W. S.; Kim, S. Y.; Lee, Y. K.; Jang, J.; Kwon, J. H., White polymeric light-emitting diodes based on doping of an orange ir complex in a fluorene blue polymer host. Mol Cryst Liq Cryst 2009, 498, 290-297.

5. Prakash, A.; Katiyar, M., White polymer light emitting diode using blend of fluorescent polymers. 16th International Workshop on Physics of Semiconductor Devices 2012, 8549.

6. Qin, L. J.; Zhu, Y. C.; Yang, H.; Ding, L.; Sun, F.; Shi, M.; Yang, S. P., White-light phosphorescence from binary coordination polymer nanoparticles. Mater Chem Phys 2013, 139, 345-349.

7. Song, H. J.; Shin, G. J.; Choi, K. H.; Lee, S.; Moon, D. K., White polymer light emitting diode materials introducing dendritic quinoxaline derivative: Synthesis, optical and electroluminescent properties. Synthetic Met 2014, 190, 1-7.

8. Sun, C.; Zhang, Y.; Sun, K.; Reckmeier, C.; Zhang, T. Q.; Zhang, X. Y.; Zhao, J.; Wu, C. F.; Yu, W. W.; Rogach, A. L., Combination of carbon dot and polymer dot phosphors for white light-emitting diodes. Nanoscale 2015, 7 , 12045-12050.

9. Taudt, C.; Baselt, T.; Oreski, G.; Hirschl, C.; Koch, E.; Hartmann, P., Cross-linking characterization of polymers based on their optical dispersion utilizing a white-light interferometer. Proc Spie 2015, 9525.

10. Roberts, R. J.; Le, D.; Leznoff, D. B., Color-tunable and white-light luminescence in lanthanide dicyanoaurate coordination polymers. Inorg Chem 2017, 56, 7948-7959.

11. Zhen, H. Y.; Xu, W.; King, W.; Chen, Q. L.; Xu, Y. H.; Jiang, J. X.; Peng, J. B.; Cao, Y., White-light emission from a single polymer with singlet and triplet chromophores on the backbone. Macromol Rapid Comm 2006, 27, 2095-2100.

12. Zhang, Y. F.; Xu, Z.; Zhang, F. J.; Wang, Y.; Zhao, S. L., White organic light emitting device with dyestuff dcjtb mended in polymer. Spectrosc Spect Anal 2008, 28, 760-762.

13. Wang, R.; Peng, J.; Qiu, F.; Yang, Y. L.; Xie, Z. Y., Simultaneous blue, green, and red emission from diblock copolymer micellar films: A new approach to white-light emission. Chem Commun 2009, 6723-6725.

14. Zhang, T. H.; Gong, Z. C.; Que, L., A white-light source operated polymer-based micromachined fabry-perot chemo/biosensor. 2009 4th leee International Conference on Nano/Micro Engineered and Molecular Systems, Vols 1 and 2 2009, 181-184. 
15. Wang, R.; Peng, J.; Qiu, F.; Yang, Y. L., Enhanced white-light emission from multiple fluorophores encapsulated in a single layer of diblock copolymer micelles. Chem Commun 2011, 47, 2787-2789

16. Willander, M.; Nur, O.; Zaman, S.; Zainelabdin, A.; Bano, N.; Hussain, I., Zinc oxide nanorods/polymer hybrid heterojunctions for white light emitting diodes. J Phys D Appl Phys 2011, 44.

17. Chen, S. A.; Chang, E. C.; Chuang, K. R.; Chao, C. I.; Wei, P. K.; Fann, W. S., Conjugated polymer blends as emitting layer for white light led. Abstr Pap Am Chem S 1998, 215, U392-U392.

18. Goustouridis, D.; Manoli, K.; Chatzandroulis, S.; Sanopoulou, M.; Raptis, I., Characterization of polymer layers for silicon micromachined bilayer chemical sensors using white light interferometry. Sensor Actuat B-Chem 2005, $111,549-554$.

19. Tian, L. L.; Zhang, W.; Yang, B.; Lu, P.; Zhang, M.; Lu, D.; Ma, Y. G.; Shen, J. C., Zinc(ii)-induced color-tunable fluorescence emission in the pi-conjugated polymers composed of the bipyridine unit: $A$ way to get white-light emission. J Phys Chem B 2005, 109, 6944-6947.

20. Huang, J. S.; Li, G.; Wu, E.; Xu, Q. F.; Yang, Y., Achieving high-efficiency polymer white-light-emitting devices. Adv Mater 2006, 18, 114-117.

21. Kassamakov, I.; Ojala, K.; Salmia, A.; Haeggstrom, E.; Aaltonen, J.; Huber, A.; Saarikko, H.; Osterberg, M.; Oinonen, M., Characterization of dents and grooves on polymer films using scanning white light interferometry. Optical Micro- and Nanometrology in Microsystems Technology 2006, 6188.

22. Kim, T. H.; Lee, H. K.; Park, O. O.; Chin, B. D.; Lee, S. H.; Kim, J. K., White-light-emitting diodes based on iridium complexes via efficient energy transfer from a conjugated polymer. Adv Funct Mater 2006, 16, 611-617.

23. Nicolai, H. T.; Hof, A. J.; Blom, P. W. M., Charge transport in white light-emitting polymers. Organic Optoelectronics and Photonics li 2006, 6192.

24. Chuang, C. Y.; Shih, P. I.; Chien, C. H.; Wu, F. I.; Shu, C. F., Bright-white light-emitting devices based on a single polymer exhibiting simultaneous blue, green, and red emissions. Macromolecules 2007, 40, 247-252.

25. Lee, P. I.; Hsu, S. L. C.; Lee, R. F., White-light-emitting diodes from single polymer systems based on polyfluorene copolymers end-capped with a dye. Polymer 2007, 48, 110-115.

26. Niu, Y. H.; Liu, M. S.; Ka, J. W.; Bardeker, J.; Zin, M. T.; Schofield, R.; Chi, Y.; Jen, A. K. Y., Crosslinkable holetransport layer on conducting polymer for high-efficiency white polymer light-emitting diodes. Adv Mater 2007, 19, $300-+$.

27. Chitara, B.; Bhat, S. V.; Vivekchand, S. R. C.; Gomathi, A.; Rao, C. N. R., White-light sources based on 
composites of gan nanocrystals with conducting polymers and nanophosphors. Solid State Commun 2008, 147, 409-413.

28. Liedtke, A.; O'Neill, M.; Wertmoller, A.; Kitney, S. P.; Kelly, S. M., White-light oleds using liquid crystal polymer networks. Chem Mater 2008, 20, 3579-3586.

29. Inoue, A.; Hosokawa, T.; Haishi, M.; Ohtani, N., 4-(dicyanomethylene)-2-methyl-6-(p-dimethylaminostyryl)-4hpyran (dcm)-doping density dependence of luminescence spectra and white emission in polymer light-emitting diodes. Physica Status Solidi C - Current Topics in Solid State Physics, Vol 6, No 1 2009, 6, 334-337.

30. Jeon, S. O.; Joo, C. W.; Yook, K.; Lee, J. Y., Color control of multilayer stacked white polymer light-emitting diodes using a quantum dot as an interlayer. Appl Phys Lett 2009, 94.

31. Cheng, G.; Fei, T.; Zhao, Y.; Ma, Y. G.; Liu, S. Y., White phosphorescent polymer light-emitting devices based on a wide band-gap polymer derived from 3,6-carbazole and tetraphenylsilane. Org Electron 2010, 11, 498-502.

32. Kim, S. H.; Jin, Y.; Yu, J. Y.; Kim, J.; Song, S.; Suh, H.; Lee, K., Color stable white polymer light-emitting diodes with single emission layer. Synthetic Met 2010, 160, 835-838.

33. Lee, P. I.; Hsu, S. L. C.; Lin, P. Y., White-light-emitting diodes from single polymer systems based on polyfluorene copolymers with quinoxaline derivatives. Macromolecules 2010, 43, 8051-8057.

34. Madhwal, D.; Rait, S. S.; Kumar, A.; Verma, A.; Tada, K.; Onoda, M.; Bhatnagar, P. K.; Mathur, P. C., Development and characterization of an efficient bio-white polymer light-emitting diode with red and green phosphorescent dyes as dopants. J Mater Sci 2010, 45, 3300-3303.

35. Hu, B.; Yao, C.; Huang, X. R., Designing of the white-light emission from a single-polymer system: Quantum theoretical study. Polym Sci Ser a+ 2011, 53, 1097-1105.

36. Lee, H. K.; Kim, T. H.; Park, J. H.; Kim, J. K.; Park, O. O., White-light-emitting diodes using miscible polymer blend doped with phosphorescent dye. Org Electron 2011, 12, 891-896.

37. Jeon, Y. P.; Choo, D. C.; Kim, T. W., Color tunable white organic light-emitting devices with a hybrid 2-methyl9,10-di(2-naphthyl)anthracene small molecule/poly(2-methoxy-5-(2-ethyhexoxy)-1,4-phenylenevinylene) polymer emitting layer. Thin Solid Films 2012, 521, 189-192.

38. Meng, L. C.; Lou, Z. D.; Yang, S. Y.; Hou, Y. B.; Teng, F.; Liu, X. J.; Li, Y. B., White organic light-emitting diodes based on a combined electromer and monomer emission in doubly-doped polymers. Chinese Phys B 2012, 21.

39. Nicolai, H. T.; Hof, A.; Blom, P. W. M., Device physics of white polymer light-emitting diodes. Adv Funct Mater 2012, 22, 2040-2047. 
40. Kim, J. H.; Song, W. S.; Yang, H., Color-converting bilayered composite plate of quantum-dot-polymer for highcolor rendering white light-emitting diode. Opt Lett 2013, 38, 2885-2888.

41. Kuo, C. P.; Chuang, C. N.; Chang, C. L.; Leung, M. K.; Lian, H. Y.; Wu, K. C. W., White-light electrofluorescence switching from electrochemically convertible yellow and blue fluorescent conjugated polymers. J Mater Chem C 2013, 1, 2121-2130.

42. Liu, B. X.; Liu, Q. Q.; Tong, C. Y.; Lu, X. D.; Lu, C. L., Blue-light-emitting surface-functionalized zns nanoparticles and their transparent polymer nanocomposites with near-white light emission. Colloid Surface $A$ 2013, 434, 213-219.

43. Wang, B. Z.; Zhang, X. P.; Liu, H. M., White-light-emitting diode based on a single-layer polymer. Aip Adv 2013, 3.

44. Chang, C. J.; Lai, C. F.; Reddy, P. M.; Chen, Y. L.; Chiou, W. Y.; Chang, S. J., Color optimization of conjugatedpolymer/ingan hybrid white light emitting diodes by incomplete energy transfer. J Lumin 2015, 160, 145-150.

45. Chen, B.; Feng, J. C., White-light-emitting polymer composite film based on carbon dots and lanthanide complexes. J Phys Chem C 2015, 119, 7865-7872.

46. Feng, C.; Sun, J. W.; Yan, P. F.; Li, Y. X.; Liu, T. Q.; Sun, Q. Y.; Li, G. M., Color-tunable and white-light emission of one-dimensional I-di-2-thenoyltartaric acid mixed-lanthanide coordination polymers. Dalton $T \mathbf{2 0 1 5}, 44,4640-$ 4647.

47. He, D. F.; Tang, Q.; Liu, S. M.; Luo, F.; Liu, Y. W.; Li, N.; Miao, J.; Wang, X. Q.; Chen, X. G.; Ma, F. J.; Liu, S. $X$., White-light emission by selectively encapsulating single lanthanide metal ions into alkaline earth metal-organic coordination polymers. Dyes Pigments 2015, 122, 317-323.

48. Leong-Hoi, A.; Claveau, R.; Flury, M.; Uhring, W.; Serio, B.; Anstotz, F.; Montgomery, P. C., Detection of defects in a transparent polymer with high resolution tomography using white light scanning interferometry and noise reduction. Proc Spie 2015, 9528.

49. Nam, G. H.; Park, I. K., Cdse quantum dot-conducting polymer hybrid structure for phosphor-free white lightemitting diodes. J Korean Phys Soc 2015, 66, 785-789.

50. da Silva, M. A. T.; Thomazini, E. F.; Albertini, M.; Renzi, W.; Franchello, F.; Dias, I. F. L.; Duarte, J. L.; Pocas, L. C.; Lourenco, S. A., Characterization of digital textile printing and polymer blend (pfo-dmp:P3ht) for application in manufacture of organic diodes emitting white light - woleds. Opt Mater 2016, 62, 119-131.

51. Fan, L. M.; Fan, W. L.; Li, B.; Zhao, X.; Zhang, X. T., W-shaped 1,3-di(2,4-dicarboxyphenyl)benzene based lanthanide coordination polymers with tunable white light emission. New J Chem 2016, 40, 10440-10446. 
